\title{
Quantum noise in a terahertz hot electron bolometer mixer
}

\author{
W. Zhang, ${ }_{1,2}^{1,2}$ P. Khosropanah, ${ }^{1}$ J. R. Gao, ${ }^{1,3, a)}$ E. L. Kollberg, ${ }^{4}$ K. S. Yngvesson, ${ }^{5}$ \\ T. Bansal, ${ }^{1,3}$ R. Barends, ${ }^{3}$ and T. M. Klapwijk ${ }^{3}$ \\ ${ }^{1}$ SRON Netherlands Institute for Space Research, Landleven 12, 9747 AD Groningen. The Netherlands \\ ${ }^{2}$ Purple Mountain Observatory, Chinese Academy of Sciences, 2 West Beijing Road, Nanjing, JiangSu 210008, \\ China \\ ${ }^{3}$ Kavli Institute of Nanoscience, Delft University of Technology, Lorentzweg 1, 2628 CJ Delft, \\ The Netherlands \\ ${ }^{4}$ Department of Microelectronics and Nanoscience, Chalmers University of Technology, \\ SE 41296 Göteborg, Sweden \\ ${ }_{5}^{5}$ Department of Electrical and Computer Engineering, University of Massachusetts, Amherst, \\ MA 01003, USA
}

(Received 8 January 2010; accepted 24 February 2010; published online 19 March 2010)

\begin{abstract}
We have measured the noise temperature of a single, sensitive superconducting $\mathrm{NbN}$ hot electron bolometer (HEB) mixer in a frequency range from 1.6 to $5.3 \mathrm{THz}$, using a setup with all the key components in vacuum. By analyzing the measured receiver noise temperature using a quantum noise (QN) model for HEB mixers, we confirm the effect of QN. The QN is found to be responsible for about half of the receiver noise at the highest frequency in our measurements. The $\beta$-factor (the quantum efficiency of the HEB) obtained experimentally agrees reasonably well with the calculated value. (C) 2010 American Institute of Physics. [doi:10.1063/1.3364936]
\end{abstract}

A superconducting hot electron bolometer (HEB) mixer, which essentially consists of a $\mathrm{NbN}$ nanobridge, metal contact pads, and an antenna structure, is the best choice for a heterodyne detector for astrophysics in the frequency range between 1.5 to $6 \mathrm{THz}$. ${ }^{1,2}$ Sensitive heterodyne spectrometers using HEBs have been realized up to $1.9 \mathrm{THz}$ for groundbased, balloon-borne, and space telescope instruments, such as the Heterodyne Instrument for Far-Infrared ${ }^{3}$ on the Herschel space telescope. To reach the ultimate receiver noise temperatures of a HEB mixer in the high end of the $\mathrm{THz}$ range $(2-6 \mathrm{THz})$, planned for future such instruments, it is crucial to understand the fundamental noise contributions from different origins. With increasing frequency, the quantum noise (QN) contribution is expected to play an increasing role. ${ }^{4}$ Here we report an experiment to demonstrate the effect of $\mathrm{QN}$ in an $\mathrm{NbN} \mathrm{HEB}$ receiver by measuring and analyzing the double sideband (DSB) receiver noise temperature $\left(T_{\mathrm{Rec}}^{\mathrm{DSB}}\right)$ in a local oscillator $(\mathrm{LO})$ frequency $\left(\mathrm{f}_{\mathrm{LO}}\right)$ range from 1.6 to $5.3 \mathrm{THz}$,

It has been well established that the classical noise sources in HEB mixer are Johnson noise and thermal fluctuation noise, ${ }^{5}$ which together contribute typically about $40 \mathrm{~K}$ at the output of an HEB. ${ }^{6}$ Callen and Welton ${ }^{7}$ showed in their generalization of the Nyquist theorem that the average energy density of an electromagnetic field, in equilibrium with an environment at a temperature $T$, includes the Planck blackbody radiation and an energy of $h f / 2$, where $f$ is the frequency. The last term represents the zero-point fluctuations of the field. ${ }^{7}$ The total power radiated into a single mode in a bandwidth $B$ can be expressed as:

$$
P_{\mathrm{CW}}(T)=\frac{h f B}{\exp (h f / k T)-1}+\frac{h f B}{2} .
$$

The first term, the Planck noise power, falls rapidly to zero at frequencies higher than $k T / h$, as the second term begins to

${ }^{\text {a)} E l e c t r o n i c ~ m a i l: ~ j . r . g a o @ t u d e l f t . n l . ~}$ dominate. This is the frequency region in which QN becomes important.

$T_{\mathrm{Rec}}^{\mathrm{DSB}}$ is measured by the Y-factor method, in which the broadband radiations from a blackbody at $295 \mathrm{~K}$ (hot) and at $77 \mathrm{~K}$ (cold) are coupled sequentially to the receiver input. Here $Y$ is the ratio of the corresponding receiver output noise powers. $T_{\mathrm{Rec}}^{\mathrm{DSB}}$ can be deduced from $T_{\mathrm{Rec}}^{\mathrm{DSB}}=\left(T_{\mathrm{eff}, \text { hot }}\right.$ $\left.-Y T_{\text {eff,cold }}\right) /(Y-1)$, where $T_{\text {eff,hot }}$ and $T_{\text {eff,cold }}$ are the equivalent temperatures of the hot/cold load, respectively, according to Eq. (1).

The theoretical model for $T_{\mathrm{Rec}}^{\mathrm{DSB}}$ of a HEB mixer, including the contribution of $\mathrm{QN}$, is based on a distributed temperature model. ${ }^{4}$ The HEB is heated by a combination of LO and direct current (DC) power, resulting in an electron temperature distribution across the bolometer, which translates into a bell-shaped resistivity profile, ${ }^{8}$ as shown in the inset of Fig. 1, for a bias point near the optimum operating region (see below). Note that the device response in this case is dominated by the center of the bolometer (the "hot spot"), indicated by the strong rise of resistivity, while outside this

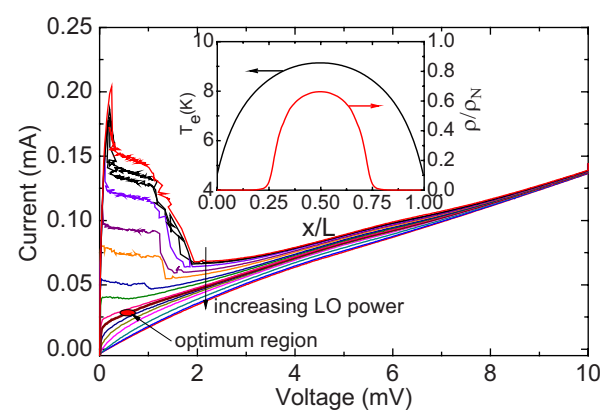

FIG. 1. (Color online) Current-voltage curves of a NbN HEB mixer taken at different LO powers, with a LO frequency of $5.3 \mathrm{THz}$, at a bath temperature of $4.2 \mathrm{~K}$, where the optimum operating region is indicated. The inset shows a distribution of the electron temperature and the normalized local resistivity calculated for the $0.2 \mu \mathrm{m}$ long $\mathrm{NbN}$ bridge (with a critical temperature of $9.3 \mathrm{~K})$. 
hotspot the device is still superconducting. $T_{\mathrm{Rec}}^{\mathrm{DSB}}$ is predicted as follows: ${ }^{4}$

$$
\begin{aligned}
T_{\mathrm{Rec}}^{\mathrm{DSB}}= & \left(L_{300}-1\right) T_{\mathrm{Planck}}(295 \mathrm{~K})+L_{300}\left(L_{4}\right. \\
& -1) T_{\mathrm{Planck}}(4 \mathrm{~K})+L_{300} L_{4} L_{\mathrm{MIX}}^{\mathrm{DSB}}\left(T_{\mathrm{CL}, \mathrm{MIX}}^{\mathrm{out}}+T_{\mathrm{IF}}\right) \\
& +\frac{h f}{2 k}\left(L_{300} L_{4} \beta-1\right),
\end{aligned}
$$

where $L_{300}$ and $L_{4}$ are the losses of the optical components that connect the superconducting bolometer to the input of the receiver. $L_{300}$ and $L_{4}$ refer to losses at room temperature and at $4 \mathrm{~K}$, respectively. $L_{\mathrm{MIX}}^{\mathrm{DSB}}$ is the conversion loss of the mixer, $T_{\text {CL,MIX }}^{\text {out }}$ the output noise due to the classical noise sources, ${ }^{5}$ and $T_{\mathrm{IF}}$ the noise temperature of the intermediate frequency (IF) amplifier chain. The last term, $(h f / 2 k)$ $\times\left(L_{300} L_{4} \beta-1\right)$, is the $\mathrm{QN}$ term $\left(T_{\mathrm{QN}}^{\mathrm{DSB}}\right)$ with a $\beta$ as the $\mathrm{QN}$ factor. The $\beta$-factor has a similar physical meaning as the quantum efficiency of any detector, and reflects the fact that in the distributed model of an HEB mixer only the central part of the bolometer (hot spot) converts the RF input signal to the IF efficiently, while the remainder of the bridge does not, leading to an increase in $\mathrm{QN}$ by a factor of $\beta$. For a perfect HEB receiver without any optical losses $\left(L_{300}=L_{4}\right.$ =1) and with $T_{\mathrm{CL}, \mathrm{MIX}}^{\mathrm{out}}+T_{\mathrm{IF}}=0, T_{\mathrm{Rec}}^{\mathrm{DSB}}$ is contributed only by QN.

For a given $\mathrm{f}_{\mathrm{LO}}$ one can experimentally determine all the parameters given in Eq. (2), except for $\beta$ and $T_{\mathrm{CL}, \mathrm{MIX}}^{\text {out }}$. To determine the contribution of $\mathrm{QN}$ one approach is to perform a series of measurements at different frequencies, preferably in the upper terahertz range, because there QN should play an increasingly important role. By fitting Eq. (2) to the experimental data one can obtain $\beta$ and $T_{\mathrm{CL}, \mathrm{MIX}}^{\mathrm{out}}$. It is generally assumed that these two parameters are determined by the operating (bias) condition of a HEB mixer, and that they are independent of $\mathrm{f}_{\mathrm{LO}}$. Earlier attempts ${ }^{9,10}$ of analyzing receiver noise temperature data at different frequencies from different HEB mixers were unable to confirm the effect of QN because the errors in the experimental data were too large to accurately determine the parameters $\beta$ and $T_{\mathrm{CL}, \mathrm{MIX}}^{\text {out }}$.

Three factors are considered to be crucial for our experiment. First, we used a spiral antenna coupled NbN HEB mixer with a $0.2 \times 2 \mu \mathrm{m}^{2} \mathrm{NbN}$ bridge, which is similar to the one described in Ref. 11. It has shown unprecedentedly high sensitivity at the upper terahertz frequencies, which is vital to exclude the contribution of any unknown effect other than $T_{\mathrm{CL}, \mathrm{MIX}}^{\text {out }}$. Second, we apply an experimental setup that is shown in Fig. 2 and that is very similar to the one used in Ref. 11. In this setup the hot/cold blackbody loads and the beam splitter are inside a vacuum enclosure. The advantages over a standard setup in air are the reduced optical loss (roughly by $3 \mathrm{~dB}$ ) and the reduced uncertainty in quantifying optical losses. The latter is critical in applying Eq. (2). All the optical losses are summarized in Table I. Third, a different measurement method ${ }^{11}$ is applied to determine $T_{\operatorname{Rec}}^{\mathrm{DSB}}$. We measure the receiver output noise power as a function of bias current $(I)$ at a fixed bias voltage, while changing the LO power (see the inset of Fig. 3). Two such traces with sufficient amount of data points are recorded, one $\left[P_{\text {out,hot }}(I)\right]$ responding to the hot load and the other $\left[P_{\text {out,cold }}(I)\right]$ to the cold load. The Y-factor can then be obtained as $Y(I)$ $=P_{\text {out,hot }}(I) / P_{\text {out,cold }}(I)$ using fitted polynomial curves to the data points. These curves are then utilized for deriving the

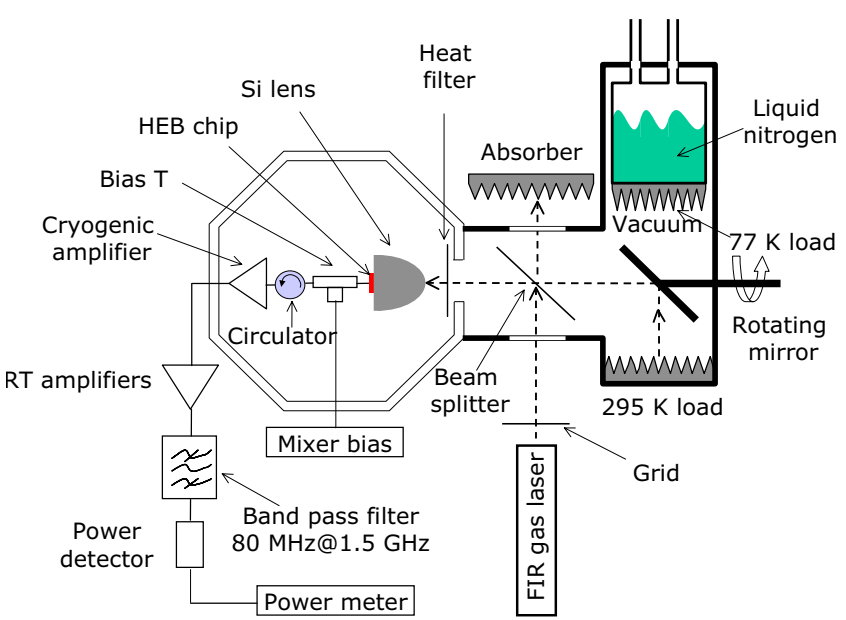

FIG. 2. (Color online) Schematic picture of the measurement setup, where the hot/cold loads and the beam splitter are built into a vacuum unit, directly attached to the HEB cryostat. Switching between the hot and cold load is done by rotating a mirror.

Y-factor and then $T_{\mathrm{Rec}}^{\mathrm{DSB}}$, using an operating point with the same value of $I$. Thanks to this method $T_{\mathrm{Rec}}^{\mathrm{DSB}}$ can be determined with an uncertainty of less than $5 \%$.

Figure 1 shows a typical set of current-voltage $(I-V)$ curves of the HEB without and with LO radiation being applied, respectively. With increasing LO power level, the curves from the top one down essentially reflect a gradual suppression of the superconductivity in the NbN bridge. The highest sensitivity is obtained at the indicated optimum operating region, where the bias voltage is around $0.6 \mathrm{mV}$, bias current $34 \mu \mathrm{A}$, and the LO power absorbed in the HEB itself is $\sim 150 \mathrm{nW}$.

Figure 3 shows the measured $T_{\mathrm{Rec}}^{\mathrm{DSB}}$ as a function of $I$ at $\mathrm{f}_{\mathrm{LO}}$ from 1.6 to $5.3 \mathrm{THz}$. We emphasize that all curves are taken at the optimum DC bias voltage of $0.6 \mathrm{mV}$ and in the same setup. The minimal $T_{\mathrm{Rec}}^{\mathrm{DSB}}$ for each $\mathrm{f}_{\mathrm{LO}}$ in Fig. 3, taken at the same bias current, is now plotted in Fig. 4, and will be used to analyze the effect of QN. The conversion loss of the mixer at different $\mathrm{f}_{\mathrm{LO}}$ is determined using the U-factor method $^{13}$ and, as summarized in Table $\mathrm{I}$, is in the range of 8.7 to $9.8 \mathrm{~dB}$.

To confirm that the physical condition of the bridge is the same at different $\mathrm{f}_{\mathrm{LO}}$, we plot all $I-V$ curves at optimum LO power in the inset of Fig. 4. They overlap well with each other. The small differences are attributed to tiny differences in actual LO power, which is difficult to adjust precisely in

TABLE I. Summary of the data for five LO frequencies $\left(f_{\mathrm{LO}}\right)$ : optical loss of the $3 \mu \mathrm{m}$ Mylar beam splitter at $300 \mathrm{~K}\left(L_{\mathrm{BS}}\right.$, calculated), heat filter at $4 \mathrm{~K}$ ( $L_{\text {filter }}$, measured), uncoated Si lens at $4 \mathrm{~K}$ ( $L_{\text {lens }}$, reflection loss calculated; absorption loss is negligible based on our measurements), coupling between antenna and HEB ( $L_{\text {coup }}$, calculated), DSB conversion loss including optical loss $\left(L_{\mathrm{Rec}}^{\mathrm{DSB}}\right.$, measured) and DSB receiver noise temperature $\left(T_{\mathrm{Rec}}^{\mathrm{DSB}}\right.$, measured).

\begin{tabular}{cllllll}
\hline \hline $\begin{array}{c}\mathrm{f}_{\mathrm{LO}} \\
(\mathrm{THz})\end{array}$ & $\begin{array}{c}L_{\mathrm{BS}} \\
(\mathrm{dB})\end{array}$ & $\begin{array}{c}L_{\text {filter }} \\
(\mathrm{dB})\end{array}$ & $\begin{array}{c}L_{\text {lens }} \\
(\mathrm{dB})\end{array}$ & $\begin{array}{c}L_{\text {coup }} \\
(\mathrm{dB})\end{array}$ & $\begin{array}{c}L_{\mathrm{Rec}}^{\mathrm{DSB}} \\
(\mathrm{dB})\end{array}$ & $\begin{array}{c}T_{\mathrm{Rec}}^{\mathrm{DSB}} \\
(\mathrm{K})\end{array}$ \\
\hline 1.63 & 0.08 & 0.9 & 1.5 & 0 & 11.95 & 842 \\
1.89 & 0.13 & 0.62 & 1.5 & 0.014 & 11.97 & 845 \\
2.52 & 0.2 & 0.73 & 1.5 & 0.067 & 12.3 & 974 \\
4.25 & 0.4 & 0.3 & 1.5 & 0.38 & 12.21 & 1193 \\
5.25 & 0.71 & 0.81 & 1.5 & 0.63 & 12.4 & 1520 \\
\hline \hline
\end{tabular}




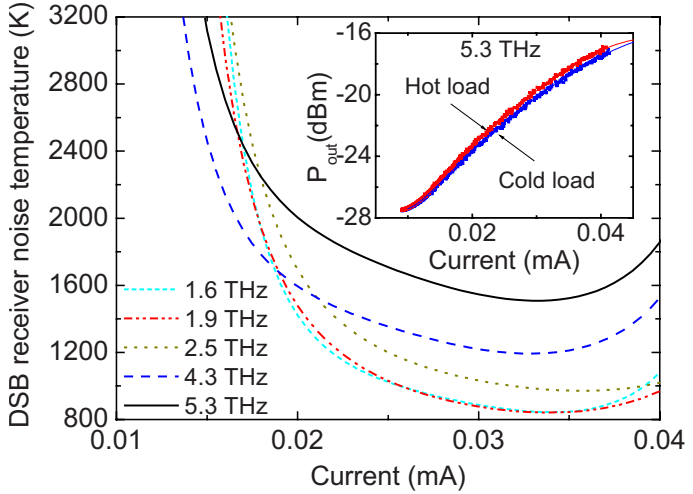

FIG. 3. (Color online) Measured DSB receiver noise temperature as a function of the current of the HEB obtained at a bias voltage of $0.6 \mathrm{mV}$ and an IF of $1.5 \mathrm{GHz}$ for five different LO frequencies. In the inset: measured receiver output powers, responding to hot and cold loads, vs current in the $\mathrm{HEB}$ at $5.3 \mathrm{THz}$ (dots) and the polynomial fit (lines).

practice. We further note that the curves of $T_{\mathrm{Rec}}^{\mathrm{DSB}}$ versus bias current in Fig. 3 are very similar in shape, with broad minima close to the same current level.

These observations provide several crucial supports for the validity of the assumptions mentioned earlier as follows: (a) The electron temperature distribution across the bridge at the optimum operating points is $f_{\mathrm{LO}}$ independent; (b) The data imply that the THz current profile along the bridge is also $f_{L O}$ independent. A recent simulation of the $\mathrm{THz}$ current distribution in HEB mixers ${ }^{12}$ also supports this point. (c) Consequently, we can also assume $\beta$ and $T_{\mathrm{CL}, \mathrm{MIX}}^{\text {out }}$ to be $\mathrm{f}_{\mathrm{LO}}$ independent.

Before applying Eq. (2), it is necessary to know the optical losses at each $\mathrm{f}_{\mathrm{LO}}$, as well as the power coupling loss $\left(L_{\text {coup }}\right)$ between the spiral antenna and the bolometer. The latter is calculated based on the impedance mismatch relation $L_{\text {coup }}^{-1}=4 R_{\mathrm{HEB}} R_{\text {antennal }}\left|R_{\mathrm{HEB}}+Z_{\text {antennal }}\right|^{-2}$, where $R_{\mathrm{HEB}}$ is the HEB impedance, taken to be $\mathrm{f}_{\mathrm{LO}}$ independent and equal to the normal state resistance, ${ }^{22}$ while $Z_{\text {antenna }}=R_{\text {antenna }}$ $+i X_{\text {antenna }}$ is the complex impedance of the antenna, simulated with 3D full-wave electromagnetic field simulation (HFSS). ${ }^{14} L_{\text {coup }}$ at different $\mathrm{f}_{\mathrm{LO}}$ are also summarized in Table I.

We can now fit Eq. (2) to the experimental data at the five $\mathrm{f}_{\mathrm{LO}}$ using $\beta$ and $T_{\text {CL.MIX }}^{\text {out }}$ as fitting parameters. The leastsquare fitted curves with three different $\beta$ values are also plotted in Fig. 4 and lead to $\beta=3.1 \pm 0.2$ and $T_{\mathrm{CL}, \mathrm{MIX}}^{\text {out }}$ $=34.5 \mathrm{~K}$. Using this $\beta$ and Eq. (2) the contribution due to only QN is then calculated and also plotted in Fig. 4 for comparison. As expected, QN plays an increasing role in $T_{\mathrm{Rec}}^{\mathrm{DSB}}$ when $\mathrm{f}_{\mathrm{LO}}$ increases. Its relative contribution to $T_{\mathrm{Rec}}^{\mathrm{DSB}}$ $\left(T_{\mathrm{QN}}^{\mathrm{DS}} / T_{\mathrm{rec}}^{\mathrm{DSB}}\right)$ increases from $20 \%$ at $1.6 \mathrm{THz}$ to $50 \%$ at 5.3 THz.

$\beta$ is estimated theoretically using Eq. 33 in Ref. 4 for a given bias current $I_{0}$ and voltage $V_{0}$ in combination with the more recent distributed electron temperature $\operatorname{model}^{8}$ (same as for the inset of Fig. 1). We find a $\beta$ of 2.3 at the optimum point $(0.6 \mathrm{mV}$ and $35 \mu \mathrm{A})$, which is a bit lower than what was found experimentally (3.1). In general, we find a reasonable agreement with regard to the absolute value. It is interesting to calculate the intrinsic noise temperature of the HEB

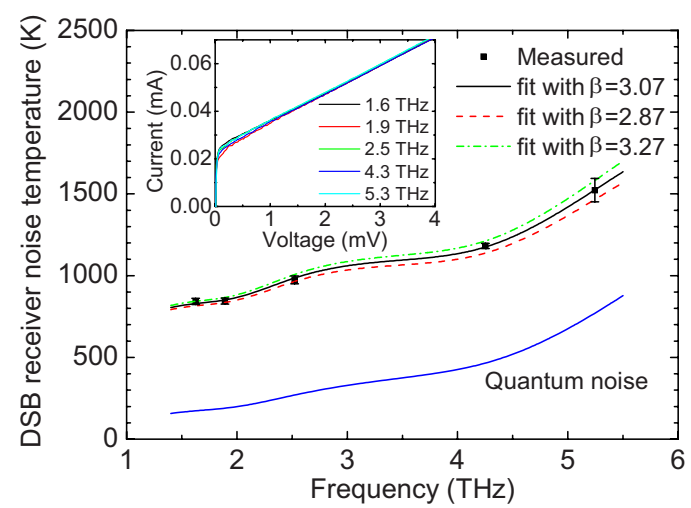

FIG. 4. (Color online) Measured minimal DSB receiver noise temperature of the HEB mixer at different LO frequencies (squares) and fitted curves for three different values of $\beta$, using Eq. (2) (lines). The noise temperature contributed by only quantum noise is also shown. The inset shows all the $I-V$ curves at optimum LO power at the five different frequencies.

mixer by itself, assuming zero optical loss and zero IF amplifier noise temperature. We find that this intrinsic mixer noise temperature at $5.25 \mathrm{THz}$ is $526 \mathrm{~K}$, or $2.1 \times h f / k$, of which $50 \%$ is due to QN.

In summary, we have demonstrated a $\mathrm{QN}$ contribution to $T_{\mathrm{Rec}}^{\mathrm{DSB}}$ in a NbN HEB heterodyne receiver and find that it increases from $20 \%$ at $1.6 \mathrm{THz}$ to $50 \%$ at $5.3 \mathrm{THz}$. To further improve the sensitivity, the challenges are to reduce the $\beta$-factor, $T_{\mathrm{CL}, \mathrm{MIX}}^{\text {out }}$, and the optical loss.

We acknowledge S.C. Shi for supporting this joint research project. The work was supported by China Exchange Programme executed by KNAW and CAS, the NSFC under Grant Nos. 10803021 and 10621303, the AMSTAR+ of RadioNet under FP7, and NWO.

${ }^{1}$ E. M. Gershenzon, G. N. Gol'tsman, I. G. Gogidze, Y. P. Gousev, A. I. Elant'ev, B. S. Karasik, and A. D. Semenov, Sov. Phys. Superconductivity. 3, 1582 (1990).

${ }^{2}$ J. Zmuidzinas and P. L. Richards, Proc. IEEE 92, 1597 (2004).

${ }^{3}$ ESA's Herschel Space Observatory, see http://sci.esa.int/science-e/www/ object/index.cfm?fobjectid $=34691$.

${ }^{4}$ E. L. Kollberg and K. S. Yngvesson, IEEE Trans. Microwave Theory Tech. 54, 2077 (2006). We use Eq. (39), but have corrected the $L_{300}$ factor in the second term and also used the notation $\beta / 2 \mathrm{G}_{\mathrm{IBBM}} \equiv L_{\mathrm{Mix}}^{\mathrm{DSB}}$ in the present paper.

${ }^{5}$ B. S. Karasik and A. I. Elantiev, Appl. Phys. Lett. 68, 853 (1996).

${ }^{6}$ H. Ekström, E. Kollberg, P. Yagoubov, G. Gol'tsman, E. Gershenzon, and S. Yngvesson, Appl. Phys. Lett. 70, 3296 (1997).

${ }^{7}$ H. B. Callen and T. A. Welton, Phys. Rev. 83, 34 (1951).

${ }^{8}$ R. Barends, M. Hajenius, J. R. Gao, and T. M. Klapwijk, Appl. Phys. Lett. 87, 263506 (2005).

${ }^{9}$ A. D. Semenov, H. Richter, H.-W. Hübers, B. Gunther, A. Smirnov, K. S. Il'in, M. Siegel, and J. P. Karamarkovic, IEEE Trans. Microwave Theory Tech. 55, 239 (2007).

${ }^{10}$ P. Khosropanah, W. Zhang, E. L. Kollberg, K. S. Yngvesson, J. R. Gao, T. Bansal, and M. Hajenius, IEEE Trans. Appl. Supercond. 19, 274 (2009).

${ }^{11}$ P. Khosropanah, J. R. Gao, W. M. Laauwen, M. Hajenius, and T. M. Klapwijk, Appl. Phys. Lett. 91, 221111 (2007).

${ }^{12}$ S. Cherednichenko, M. Kroug, H. Merkel, P. Khosropanah, A. Adam, E. Kollberg, D. Loudkov, G. Gol'tsman, B. Voronov, H. Richter, and H.-W. Hübers, Physica C 372-376, 427 (2002).

${ }^{13}$ E. L. Kollberg, K. S. Yngvesson, Y. Ren, W. Zhang, P. Khosropanah, and J. R. Gao (unpublished).

${ }^{14} 3 \mathrm{D}$ Full-Wave Electromagnetic Field Simulation, see http:// www.ansoft.com/products/hf/hfss/. 